\title{
Change in soluble epoxide hydrolase (sEH) during cisplatin-induced acute renal failure in mice
}

\author{
Terumasa Hashimoto, Yang-II Fang, Hisayuki Ohata and Kazuo Honda \\ Division of Pharmacology, Department of Pharmacology, Toxicology \& Therapeutics, School of Pharmacy, Showa University, \\ 1-5-8 Hatanodai, Shinagawa-ku, Tokyo 142-8555, Japan
}

(Received February 20, 2015; Accepted April 14, 2015)

\begin{abstract}
Cisplatin is one of the most effective chemotherapeutic agents against various types of cancers; however, it is also associated with nephrotoxicity. Recently, it was reported that inflammatory mechanisms play a key role in the development of nephrotoxicity. Epoxyeicosatrienoic acids (EETs) have an anti-inflammatory effect and are metabolized by soluble epoxide hydrolase (sEH: encoded by EPHX2 gene). Here, we determined the change in sEH activity and EPHX2 expression in renal tissue associated with the development of cisplatin-induced nephrotoxicity. Cisplatin administration decreased hydrolase activity accompanied by down-regulation of sEH and EPHX2 expression. The down-regulation occurred prior to the elevation of blood urea nitrogen (BUN) and tumor necrosis factor- $\alpha$ (TNF- $\alpha$ ) gene expression or at treatment with low dose cisplatin. In addition, a negative correlation was found between EPHX2 expression and renal thiobarbituric acid reactive substance (TBARS), and edaravone, a radical scavenger, administration did not down-regulate expression of this gene. The results of this study suggest that cisplatin decreased sEH activity through the down-regulation of sEH and EPHX2 expression, and this downregulation was involved in a negative feedback loop to protect renal tissue from further damage. Thus, $\mathrm{sEH}$ is a potential therapeutic target of cisplatin-induced nephrotoxicity.
\end{abstract}

Key words: Cisplatin, Nephrotoxicity, Soluble epoxide hydrolase, Epoxyeicosatrienoic acid

\section{INTRODUCTION}

Cisplatin is a widely used chemotherapeutic agent; however, its clinical use is sometimes limited by adverse effects such as nephrotoxicity (Madias and Harrington, 1978). Although the mechanisms underlying cisplatininduced acute nephrotoxicity are not fully understood, it was reported that the induction of reactive oxygen species (ROS) contribute to the development of nephrotoxicity (Hannemann and Baumann, 1988). Radical scavengers protect against cisplatin-induced nephrotoxicity (Sueishi et al., 2002). Some transcription factors involved in gene expression are regulated by ROS (Schreck et al., 1992; Simon et al., 1998). Activation of these signaling pathways and the corresponding induction of proinflammatory cytokines is responsible for cisplatin-induced nephrotoxicity.

ROS also activates phospholipase $\mathrm{A}_{2}$, resulting in the stimulation of arachidonic acid (AA) (Xu et al., 2003). Epoxyeicosatrienoic acids (EETs) are metabolites of AA and have multiple functions including vasodilation, anti-inflammatory effects, angiogenesis and mitogenesis (Spector and Norris, 2007). EETs are converted to dihydroxyeicosatrienoic acids (DHETs) by soluble epoxide hydrolase (sEH: encoded by the EPHX2 gene). A recent study indicated that genetic disruption of EPHX2 attenuated the cisplatin-induced increase of blood urea nitrogen (BUN) and histological changes of renal damage (Liu et al., 2012). Thus, EETs and sEH might be a novel therapeutic target. However, few studies have investigated changes in sEH activity or EPHX2 expression associated with the development of nephrotoxicity induced by cisplatin administration.

The aim of the present study was to measure changes in sEH activity and EPHX2 expression in renal tissues associated with the development of cisplatin-induced nephrotoxicity. This investigation revealed oxidative stress-dependent EPHX2 down regulation and decreased sEH activity during cisplatin-induced nephrotoxicity. This phenomenon might be a negative feedback loop that contributes to oxidative stress.

Correspondence: Terumasa Hashimoto (E-mail: t-hashi@pharm.showa-u.ac.jp) 


\section{MATERIALS AND METHODS}

\section{Animals}

All experiments were conducted in accordance with the regulations of the Committee of Animal Care and Welfare of Showa University. Male ddY mice weighing 35-45 g (Tokyo Jikken, Tokyo, Japan) were maintained in an air-conditioned room at $20 \pm 2{ }^{\circ} \mathrm{C}$, with $50 \pm 20 \%$ relative humidity and a $12 \mathrm{hr}$ light-dark cycle (lights on 8:0020:00). Animals received a standard laboratory diet and water was provided ad libitum.

\section{Experimental procedures}

Animals were anesthetized with 5\% isoflurane (Escain $^{\circledR}$; Mylan, Tokyo, Japan), and anesthesia was maintained with $1.0-1.5 \%$ isoflurane. Under anesthesia, 10 or $20 \mathrm{mg} / \mathrm{kg}$ of cisplatin (Wako Pure Chemical, Osaka, Japan) was intravenously infused over 15 min interval using a syringe pump (KDS200P, Muromachi Kikai, Tokyo, Japan). Three days following cisplatin administration, blood samples were obtained for the determination of BUN or serum creatinine $(\mathrm{sCr})$. The kidney was perfused with saline and isolated to measure sEH activity and thiobarbituric acid reactive substance (TBARS) and for real-time reverse transcription polymerase chain reaction (real-time RT-PCR) analysis. Edaravone (Radicut ${ }^{\circledR}$; Mitsubishi Tanabe, Tokyo, Japan) was administered intravenously at a dose of $10 \mathrm{mg} / \mathrm{kg}$ just before cisplatin administration and once a day for 2 days.

\section{Real-time RT-PCR}

Total RNA was extracted from renal tissue using TRIzol reagent (Invitrogen, Carlsbad, CA, USA). Samples were evaluated spectrophotometrically at $260 \mathrm{~nm}$ to determine concentration. Overall, $1 \mu \mathrm{g}$ of total RNA was subjected to a reverse transcription reaction using a SUPERSCRIPT first-strand cDNA synthesis system (Invitrogen). Target cDNA levels were quantified by real-time RT-PCR using the ABI PRISM 7000 Sequence Detector (Applied Biosystems, Foster City, CA, USA) and SYBR GreenER qPCR SuperMix (Invitrogen) in accordance with the manufacturer's instructions.

The forward primer for $\beta$-actin was 5'-CCTTCCTTCTTGGGTATGGAATC-3' and the reverse primer was 5'-TGCTAGGAGCCAGAGCAGTAATC-3'. Other primers were purchased from Qiagen (Valencia, CA, USA).

\section{Immunofluorescence labeling}

Renal tissues were fixed in $4 \%$ paraformaldehyde and incubated in $20 \%$ sucrose for 2 days. Frozen sections
(7 $\mu \mathrm{m})$ were blocked with 5\% normal goat serum for $60 \mathrm{~min}$. Sections were incubated with anti-sEH polyclonal antibody (Santa Cruz Biotechnology, Santa Cruz, CA, USA) for $12 \mathrm{hr}$ at $4{ }^{\circ} \mathrm{C}$. After washing in PBS, the sections were incubated in TRITC-conjugated anti-IgG secondary antibody (Sigma, St Louis, MO, USA) for $120 \mathrm{~min}$ at room temperature. Fluorescent images were acquired by confocal microscopy (LSM510. Carl Zeiss, Oberkochen, Germany).

\section{Determination of AA metabolites}

To measure sEH activity in renal tissue, tissue was homogenized with ice-cold ethanol. The supernatant obtained from tissue homogenate was acidified by $1 \mathrm{~N}$ $\mathrm{HCl}$ and passed through a Sep-Pak $\mathrm{C}_{18}$ cartridge (Waters Co. Ltd., Milford, MA, USA). The retained AA metabolites were eluted with $3 \mathrm{~mL}$ of ethyl acetate-methanol (9:1). An internal standard (100 pg of $\mathrm{PGB}_{2}$ ) was added to the sample before passage through the cartridge. The sample was evaporated by vacuum concentration (5305, Eppendorf AG, Hamburg, Germany). Then, AA metabolites were dissolved in $1 \mathrm{~mL}$ of mobile phase (ion-exchanged water/acetonitrile/formic acid $=63: 37: 0.1$, $\mathrm{v} / \mathrm{v} / \mathrm{v}$ ), and injected into the liquid chromatography-tandem mass spectrometry (LC-MS/MS) system. Mass spectrometric analyses was performed by high performance liquid chromatography (Shimazu Co. Ltd., Kyoto, Japan) coupled with a linear ion trap quadrupole mass spectrometer (QTRAP5500, AB Sciex, Framingham, MA, USA) as described previously (Kuwata et al., 2014).

\section{Renal function and oxidative stress}

Renal function was assessed by measurement of BUN and $\mathrm{sCr}$ using commercially available assay kits (Wako Pure Chemical). Oxidative stress was assessed by measurement of TBARS using a TBARS assay kit (Cayaman Chemical, Ann Arbor, MI, USA).

\section{Data analysis}

sEH activity in renal tissue was expressed as a ratio of 11, 12-EET and 11, 12-DHET. Gene expression was normalized to $\beta$-actin from the same sample of cDNA. All values were represented as the mean \pm S.E. Student's $t$-test or Dunnett's multiple comparison test was used to determine the statistical differences between the means of test and control groups. A $P$-value less than 0.05 was considered significant. 
Change in sEH with cisplatin-induced nephrotoxicity

\section{RESULTS}

\section{Change in renal function, sEH activity and TBARS}

Table 1 shows changes in renal function, sEH activity and TBARS. Renal function significantly deteriorated after cisplatin administration (saline group vs cisplatin group: BUN, $25.7 \pm 1.11 \mathrm{mg} / \mathrm{dL}$ vs $80.1 \pm 14.6 \mathrm{mg} / \mathrm{dL}$, $P<0.01 ; \mathrm{sCr}, 0.45 \pm 0.01 \mathrm{mg} / \mathrm{dL}$ vs $0.91 \pm 0.19 \mathrm{mg} / \mathrm{dL}$, $P<0.05)$. Cisplatin also induced oxidative stress. TBARS in the cisplatin group $(3.34 \pm 0.29 \mathrm{nmol} / \mathrm{mg}$ protein $)$ was significantly higher $(P<0.01)$ than in the saline group $(2.24 \pm 0.16 \mathrm{nmol} / \mathrm{mg}$ protein). However, hydrolase activity in renal tissue decreased following cisplatin administration. Statistically significant differences were observed between cisplatin and the saline group.

\section{Expression of sEH and EPHX2}

The expression of sEH in renal tissue was investigated by immunofluorescence (Fig. 1A). Confocal microscopy demonstrated the expression of sEH in proximal tubules under normal condition, but cisplatin administration down-regulated sEH levels. In addition, quantitative PCR analysis indicated that expression of EPHX2 in the cisplatin group was significantly reduced compared with the saline group $(P<0.01)$ (Fig. 1B).

\section{Implication of oxidative stress on EPHX2 expression}

Fig. 2A illustrates the correlation between EPHX2 expression and TBARS in renal tissues. A negative correlation was found between $E P H X 2$ expression and TBARS

Table 1. Change in renal function, sEH activity and TBARS in cisplatin-induced nephrotoxicity in mice.

\begin{tabular}{lcccc}
\hline & BUN $(\mathrm{mg} / \mathrm{dL})$ & $\mathrm{sCr}(\mathrm{mg} / \mathrm{dL})$ & TBRAS $(\mathrm{nmol} / \mathrm{mg}$ protein) & sEH (EET/DHET) \\
\hline Saline & $25.7 \pm 1.11$ & $0.45 \pm 0.55$ & $2.08 \pm 0.16$ & $0.09 \pm 0.02$ \\
Cisplatin & $80.1 \pm 14.8^{* *}$ & $0.90 \pm 0.19^{*}$ & $3.34 \pm 0.29^{*}$ & $0.14 \pm 0.03^{* *}$ \\
\hline
\end{tabular}

Acute renal failure was induced by a single intravenous administration of cisplatin $(20 \mathrm{mg} / \mathrm{kg}) . * \mathrm{P}<0.05, * * \mathrm{P}<0.01$ : Statistically significant difference from saline group. $\mathrm{N}=10-13$.

A)

\section{Saline}
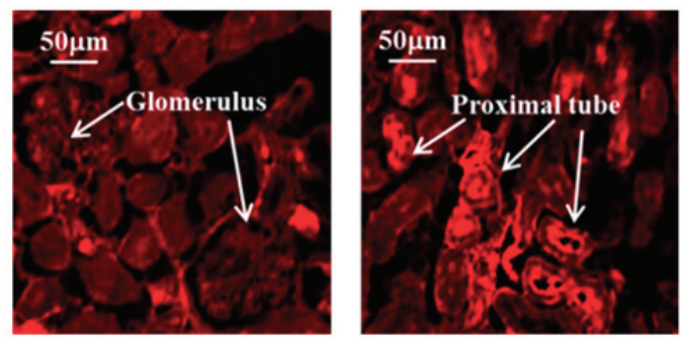

\section{Cisplatin}

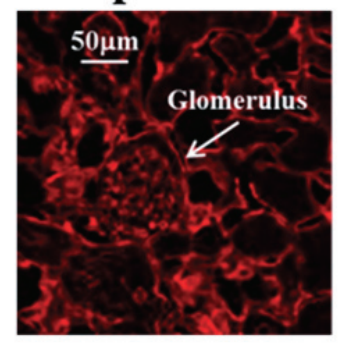

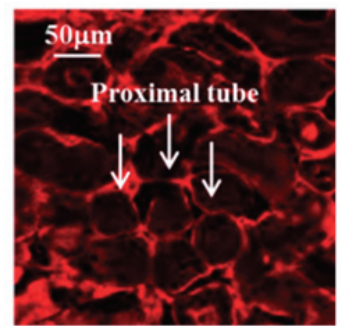

B)

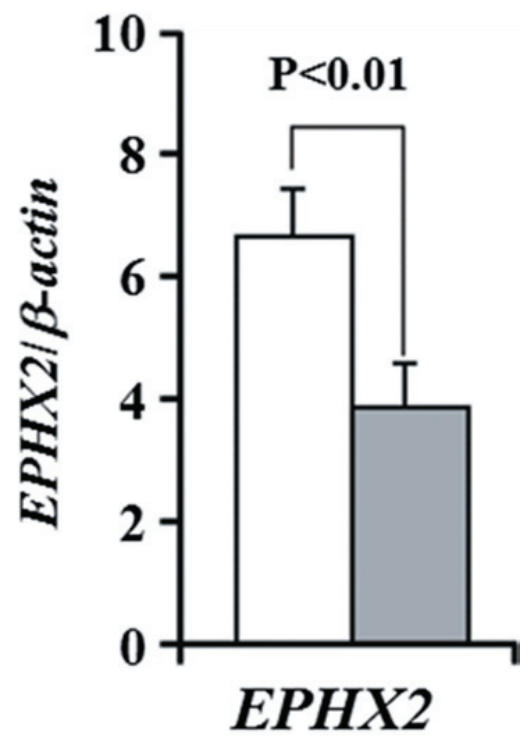

Fig. 1. Changes in $\mathrm{sEH}$ and $E P H X 2$ expression in cisplatin-induced nephrotoxicity in mice. Acute renal failure was induced by a single intravenous administration of cisplatin $(20 \mathrm{mg} / \mathrm{kg})$. A) Distribution of $\mathrm{sEH}$ or B) EPHX2 expression in renal tissues from cisplatin and saline-treated animals. mRNA levels were determined by quantitative PCR and normalized to $\beta$-actin. Each column represents the mean \pm S.E. of 10 animals. $\square$ : Saline; $\mathbf{a}$ : Cisplatin. 
A)

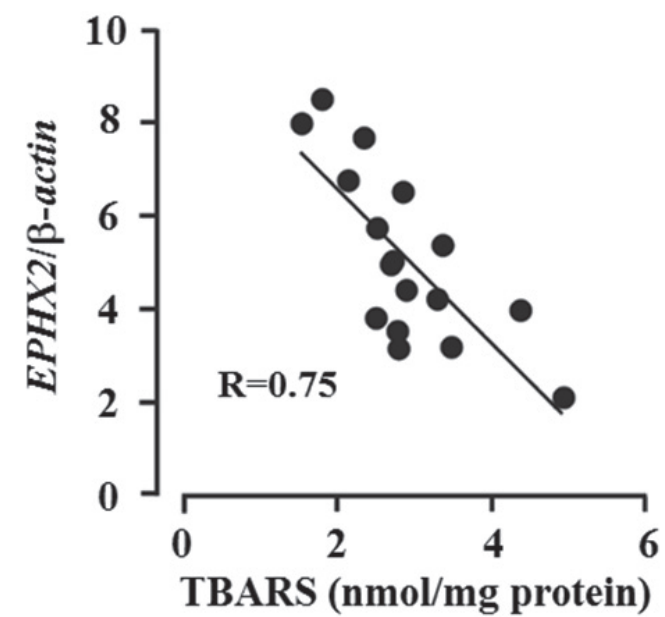

B)
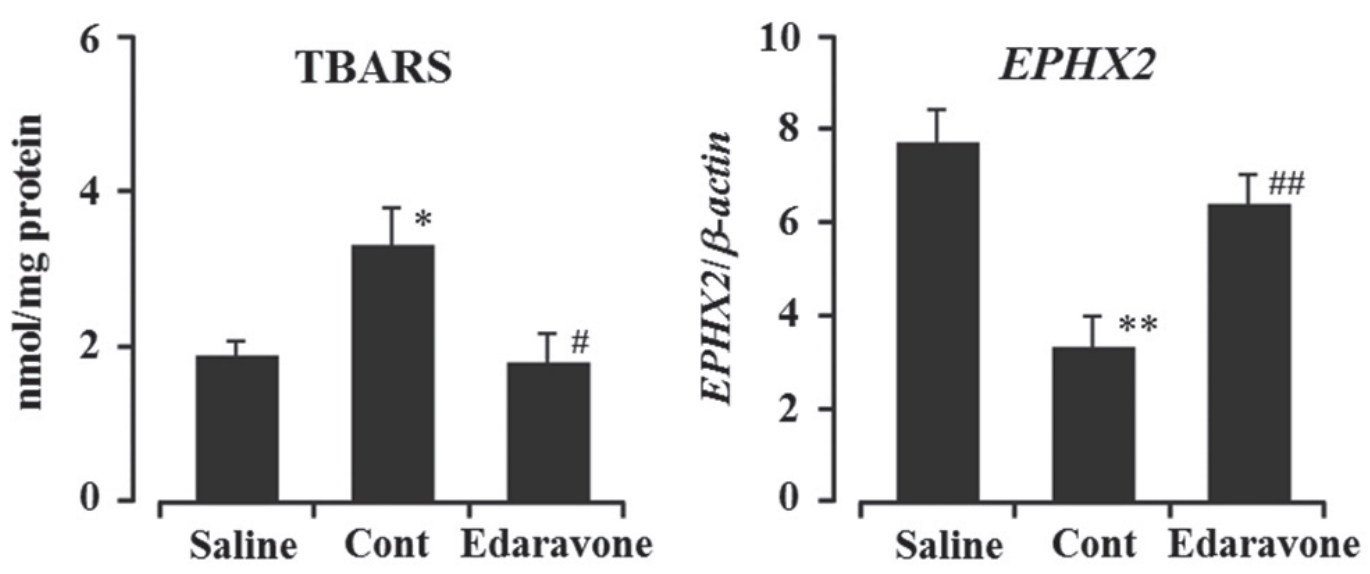

Fig. 2. Implication of oxidative stress on $E P H X 2$ expression. A) Correlation of $E P H X 2$ expression and TBARS in renal tissue. Data was obtained from saline-, 10 and $20 \mathrm{mg} / \mathrm{kg}$ cisplatin-treated groups. B) Effect of edaravone on EPHX2 expression and TBARS in renal tissue. Acute renal failure was induced by a single intravenous administration of cisplatin $(20 \mathrm{mg} / \mathrm{kg})$. Edaravone was administered intravenously at a dose of $10 \mathrm{mg} / \mathrm{kg}$ before cisplatin administration and once a day for 2 days. Each column represents the mean \pm S.E. of $4-6$ animals. ${ }^{*} \mathrm{P}<0.05, * * \mathrm{P}<0.01$ : Statistically significant difference from saline group. ${ }^{\#} \mathrm{P}<0.05,{ }^{\# \mathrm{P}}<0.01$ : Statistically significant difference from control group.

with a correlation coefficient of 0.75 . Subsequently, we investigated the effect of edaravone, a radical scavenger, on EPHX2 expression and TBARS in renal tissues (Fig. 2B). Cisplatin at $20 \mathrm{mg} / \mathrm{kg}$ produced a significant increase of TBARS compared with the saline group $(\mathrm{P}<0.05)$. Treatment with $10 \mathrm{mg} / \mathrm{kg}$ of edaravone reduced this increase and, so, statistically significant differences were observed between the edaravone-treated and the control group (edaravone-treated vs control: $1.73 \pm 0.39 \mathrm{nmol} / \mathrm{mg}$ protein vs $3.24 \pm 0.68, P<0.05$ ). Expression of EPHX2 significantly decreased following administration of cisplatin at $20 \mathrm{mg} / \mathrm{kg}(\mathrm{P}<0.01)$.
This down-regulation of $E P H X 2$ was also attenuated by $10 \mathrm{mg} / \mathrm{kg}$ of edaravone. There were significant differences between the edaravone-treated and control groups $(P<0.01)$.

\section{Time course change in renal function, EPHX2 and TNF- $\alpha$ expression}

Next, we examined the time course change in renal function, and EPHX2 and TNF- $\alpha$ expression after cisplatin administration. Increased BUN was not evident until 3 days after $20 \mathrm{mg} / \mathrm{kg}$ of cisplatin administration, which also increased $T N F-\alpha$ expression within 2 days of admin- 
Change in sEH with cisplatin-induced nephrotoxicity

istration. In contrast, down-regulation of EPHX2 was observed as early as 1 day after cisplatin administration $(P<0.05)$. Following $10 \mathrm{mg} / \mathrm{kg}$ of cisplatin administration, EPHX2 expression became gradually lower, and there were significant differences when comparing day 0 with days $2(P<0.05)$ and $3(P<0.01)$ days after cisplatin administration (Fig. 3).

\section{DISCUSSION}

An early response to cisplatin-induced nephrotoxicity includes the activation of signaling pathways such as NF-кB (Lee et al., 2006) or p38 MAPK (Luo et al., 2008) and the induction of cytokine or adhesion molecules. EETs produce an anti-inflammatory effect by inhibiting $\mathrm{NF}-\kappa \mathrm{B}$ transcription and EET analogs attenuate cisplatin-induced nephrotoxicity by reducing inflammation and apoptosis (Khan et al., 2013). Previous studies indicated the level of EETs in renal tissue might play a key role
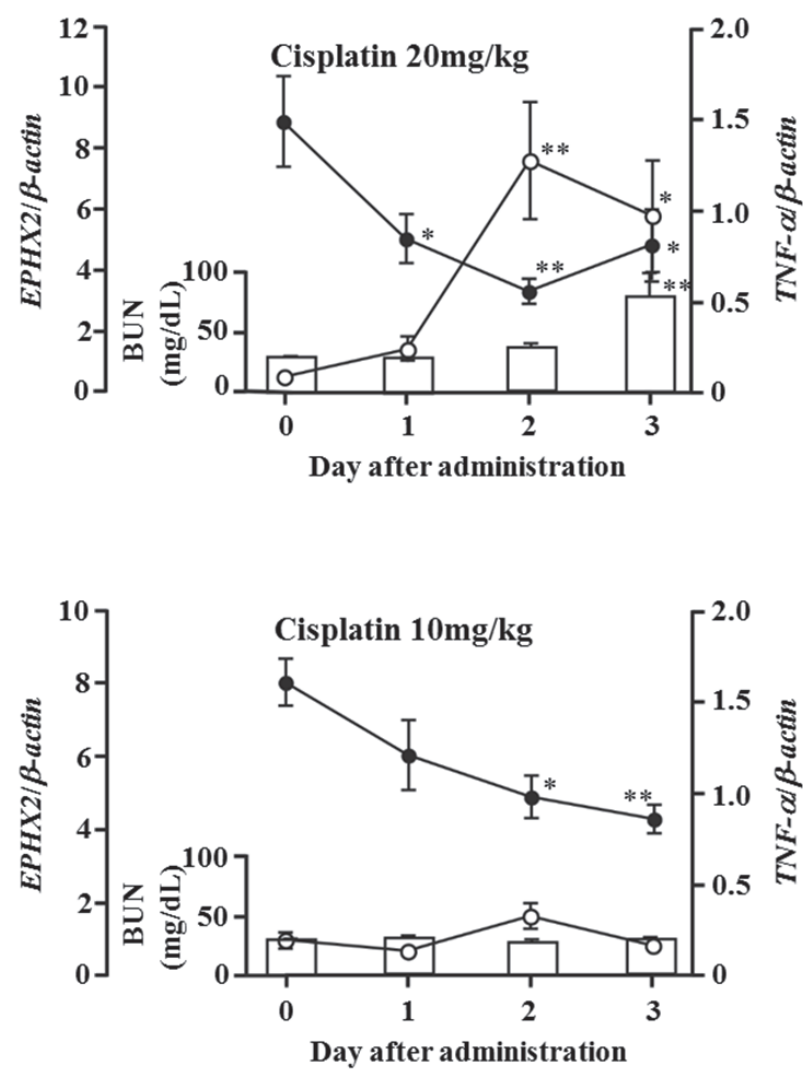

Fig. 3. Time course of changes of renal function, EPHX2 and $T N F-\alpha$ expression after cisplatin administration. Each data point represents the mean \pm S.E. of 7 animals. ${ }^{*} P<0.05,{ }^{*} P<0.01$; statistically significant difference compared with day 0 . $\square$ : BUN; $\bullet: E P H X 2 ; \circ: T N F-\alpha$. in the development of cisplatin-induced nephrotoxicity. EETs are produced by cytochrome P450, and catalyzed by $\mathrm{sEH}$, and these are critical regulatory points for EETs production. The most notable and intriguing findings of the present investigation are that cisplatin decreased $\mathrm{sEH}$ activity through the down-regulation of sEH and EPHX2 expression. The involvement of oxidative stress in the down-regulation of EPHX2 was investigated by administering edaravone. A negative correlation was observed between EPHX2 expression and TBARS. In addition, the down-regulation of $E P H X 2$ was inhibited by edaravone treatment. A previous study revealed that $\mathrm{sEH}$ expression in Hep 3B cells was suppressed by high glucose, and that the inhibition of ROS production attenuated this suppression (Oguro et al., 2009). Although little is known about its transcriptional regulation of $E P H X 2$, these findings indicate that the down-regulation of EPHX2 is induced in an oxidative stress-dependent manner.

Expression of $E P H X 2$ was significantly reduced by cisplatin administration. It was also reported that ischemiareperfusion injury in kidney down-regulated $\mathrm{sEH}$ (Lee et al., 2012). There is a discrepancy between renal function and expression of $E P H X 2$ because, the down-regulation of EPHX2 induces the increase of EETs level in renal tissue and results in renal protective effect. To explain this discrepancy, we examined the time course change in renal function, and $E P H X 2$ and $T N F-\alpha$ expression after cisplatin administration. The production of TNF- $\alpha$ in response to cisplatin is a central element in nephrotoxicity (Miller et al., 2010). In the present study, BUN was increased at 3 days and the up-regulation of $T N F-\alpha$ started from 2 days after $20 \mathrm{mg} / \mathrm{kg}$ cisplatin administration. However, the down-regulation of EPHX2 occurred from 1 day after $20 \mathrm{mg} / \mathrm{kg}$ of cisplatin administration. There is a lag between down-regulation of EPHX2 and development of nephrotoxicity. In the case of $10 \mathrm{mg} / \mathrm{kg}$ cisplatin, significant down-regulation of EPHX2 was observed at 2 and 3 days after administration: but, changes of BUN and $T N F-\alpha$ expression were not observed. As we described previously, the genetic disruption of EPHX2 attenuates cisplatin-induced nephrotoxicity (Liu et al., 2012). Therefore, we speculate this oxidative stress-dependent downregulation of sEH and $E P H X 2$, consequently increased the levels of EETs, which is a negative feedback loop (Fig. 4) and a vital mechanism to protect the renal tissue from further damage.

Several biomarkers are used as indicators of kidney injury including $\mathrm{BUN}, \mathrm{sCr}$ and urinary $\mathrm{N}$-acetyl- $\beta$-Dglucosaminidase. However, traditional biomarkers are not sensitive for the early detection of renal damage. Recently, L-type fatty acid binding protein (L-FABP) and kid- 


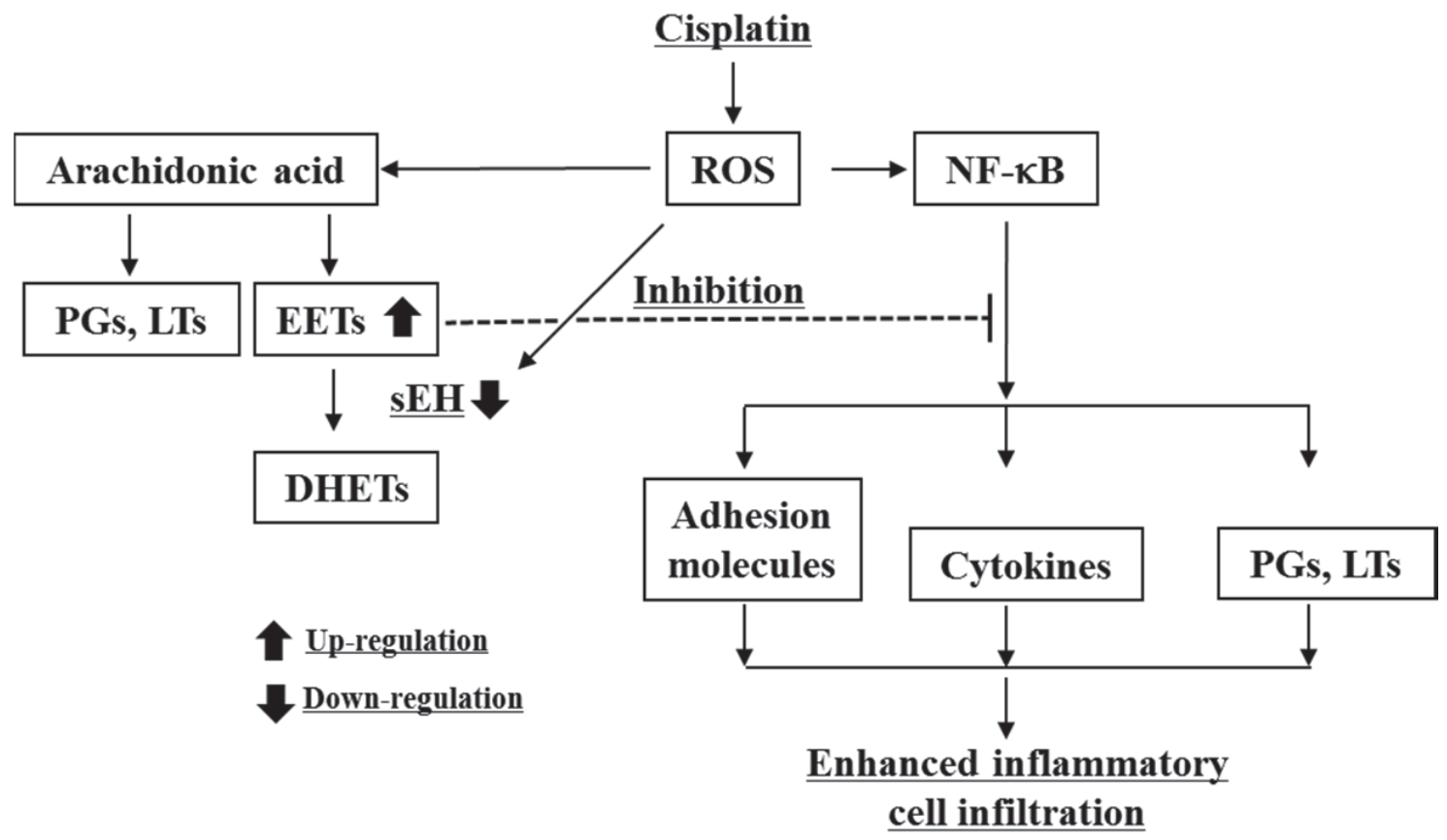

Fig. 4. Schematic diagram of the negative feedback loop accompanied by down-regulation of sEH and EPHX2 expression by cisplatin-induced nephrotoxicity.

ney injury molecule-1 (Kim-1) were suggested to be useful biomarkers for the early detection of renal damage (Nakamura et al., 2008; Ichimura et al., 2004). In cisplatin-induced nephrotoxicity, urinary L-FABP levels increased prior to the elevation of BUN (Negishi et al., 2009). Although sCr does not increase by treatment with low dose cisplatin $(5 \mathrm{mg} / \mathrm{kg})$, urinary Kim-1 significantly increases its levels (Hosohata et al., 2012). The downregulation occurred prior to the elevation of BUN and $T N F-\alpha$ gene expression or at treatment with low dose cisplatin. Thus, sEH encoded by the EPHX2 gene might be useful for the screening of nephrotoxicity. Further studies are needed to clarify its potential use as a biomarker.

Taken together, this investigation revealed the oxidative stress-dependent EPHX2 down-regulation and subsequent decrease of sEH activity was associated with the development of cisplatin-induced nephrotoxicity. Moreover, the expression of $E P H X 2$ was lowered prior to the elevation of BUN and TNF- $\alpha$ expression. This phenomenon might be a negative feedback loop to protect renal tissue from further damage. These results indicate $\mathrm{sEH}$ inhibition might represent a novel approach for the treatment of cisplatin-induced nephrotoxicity.

\section{ACKOWLEDGEMENTS}

This work was supported in part by a Showa University Special Grant-in-Aid for Innovative Collaborative Research Project and a Special Research Grant-in-Aid for Development of Characteristic Education from the Japanese Ministry of Education, Culture, Sports, Science and Technology. The funding bodies had no role in the study design, data collection or analysis, writing of the report, or the decision to submit the article for publication.

Conflict of interest---- The authors declare that there is no conflict of interest.

\section{REFERENCES}

Hannemann, J. and Baumann, K. (1988): Cisplatin-induced lipid peroxidation and decrease of gluconeogenesis in rat kidney cortex: different effects of antioxidants and radical scavengers. Toxicology, 51, 119-132.

Hosohata, K., Ando, H. and Fujimura, A. (2012): Urinary vanin-1 as a novel biomarker for early detection of drug-induced acute kidney injury. J. Pharmacol. Exp. Ther., 341, 656-662.

Ichimura, T., Hung, C.C., Yang, S.A., Stevens, J.L. and Bonventre, J.V. (2004): Kidney injury molecule-1: a tissue and urinary biomarker for nephrotoxicant-induced renal injury. Am. J. Physiol. Renal. Physiol., 286, F552-563. 
Change in sEH with cisplatin-induced nephrotoxicity

Khan, M.A., Liu, J., Kumar, G., Skapek, S.X., Falck, J.R. and Imig, J.D. (2013): Novel orally active epoxyeicosatrienoic acid (EET) analogs attenuate cisplatin nephrotoxicity. FASEB J., 27, 29462956.

Kuwata, H., Yoshimura, M., Sasaki, Y., Yoda, E., Nakatani, Y., Kudo, I. and Hara, S. (2014): Role of long-chain acyl-coenzyme A synthetases in the regulation of arachidonic acid metabolism in interleukin $1 \beta$-stimulated rat fibroblasts. Biochim. Biophys. Acta., 1841, 44-53.

Lee, J.P., Yang, S.H., Lee, H.Y., Kim, B., Cho, J.Y., Paik, J.H., Oh, Y.J., Kim, D.K., Lim, C.S. and Kim, Y.S. (2012): Soluble epoxide hydrolase activity determines the severity of ischemia-reperfusion injury in kidney. PLoS One, 7, e37075.

Lee, S., Moon, S.O., Kim, W., Sung, M.J., Kim, D.H., Kang, K.P., Jang, Y.B., Lee, J.E., Jang, K.Y., Lee, S.Y. and Park, S.K. (2006): Protective role of L-2-oxothiazolidine-4-carboxylic acid in cisplatin-induced renal injury. Nephrol. Dial. Transplant., 21, 20852095.

Liu, Y., Webb, H.K., Fukushima, H., Micheli, J., Markova, S., Olson, J.L. and Kroetz, D.L. (2012): Attenuation of cisplatininduced renal injury by inhibition of soluble epoxide hydrolase involves nuclear factor $\kappa \mathrm{B}$ signaling. J. Pharmacol. Exp. Ther., 341, 725-734.

Luo, J., Tsuji, T., Yasuda, H., Sun, Y., Fujigaki, Y. and Hishida, A. (2008): The molecular mechanisms of the attenuation of cisplatin-induced acute renal failure by $\mathrm{N}$-acetylcysteine in rats. Nephrol. Dial. Transplant., 23, 2198-2205.

Madias, N.E. and Harrington, J.T. (1978): Platinum nephrotoxicity. Am. J. Med., 65, 307-314.

Miller, R.P., Tadagavadi, R.K., Ramesh, G. and Reeves, W.B. (2010): Mechanisms of cisplatin nephrotoxicity. Toxins, 2, 2490-
2518.

Nakamura, K., Ito, K., Kato, Y., Sugaya, T., Kubo, Y. and Tsuji, A. (2008): L-type fatty acid binding protein transgenic mouse as a novel tool to explore cytotoxicity to renal proximal tubules. Drug Metab. Pharmacokinet., 23, 271-278.

Negishi, K., Noiri, E., Doi, K., Maeda-Mamiya, R., Sugaya, T., Portilla, D. and Fujita, T. (2009): Monitoring of urinary L-type fatty acid-binding protein predicts histological severity of acute kidney injury. Am. J. Pathol., 174, 1203-1211.

Oguro, A., Fujita, N. and Imaoka, S. (2009): Regulation of soluble epoxide hydrolase (sEH) in mice with diabetes: high glucose suppresses sEH expression. Drug Metab. Pharmacokinet., 24, 438-445.

Schreck, R., Albermann, K. and Baeuerle, P.A. (1992): Nuclear factor kappa B: an oxidative stress-responsive transcription factor of eukaryotic cells (a review). Free Radic. Res. Commun., 17, 221-237.

Simon, A.R., Rai, U., Fanburg, B.L. and Cochran, B.H. (1998): Activation of the JAK-STAT pathway by reactive oxygen species. Am. J. Physiol., 275, C1640-1652.

Spector, A.A. and Norris, A.W. (2007): Action of epoxyeicosatrienoic acids on cellular function. Am. J. Physiol. Cell Physiol., 292, C996-1012.

Sueishi, K., Mishima, K., Makino, K., Itoh, Y., Tsuruya, K., Hirakata, H. and Oishi, R. (2002): Protection by a radical scavenger edaravone against cisplatin-induced nephrotoxicity in rats. Eur. J. Pharmacol., 451, 203-208.

Xu, J., Yu, S., Sun, A.Y. and Sun, G.Y. (2003): Oxidant-mediated AA release from astrocytes involves CPLA(2) and iPLA(2). Free Radic. Biol. Med., 34, 1531-1543. 